University of Nebraska - Lincoln

DigitalCommons@University of Nebraska - Lincoln

7-2009

\title{
Spectroscopic investigations on Polypropylene-Carbon Nanofiber Composites: I. Raman and Electron Spin Resonance Spectroscopy
}

\author{
Mircea Chipara \\ University of Texas Pan American, mchipara@utpa.edu \\ John R. Villarreal \\ University of Texas Pan American \\ Magdalena Dorina Chipara \\ University of Texas Pan American, dorina.chipara@utrgv.edu \\ Karen Lozano \\ University of Texas Pan American \\ Alin Cristian Chipara \\ University of Texas Pan American, cristian.chipara@gmail.com \\ See next page for additional authors
}

Follow this and additional works at: https://digitalcommons.unl.edu/physicssellmyer

Part of the Physics Commons

Chipara, Mircea; Villarreal, John R.; Chipara, Magdalena Dorina; Lozano, Karen; Chipara, Alin Cristian; and Sellmyer, David J., "Spectroscopic investigations on Polypropylene-Carbon Nanofiber Composites: I. Raman and Electron Spin Resonance Spectroscopy" (2009). David Sellmyer Publications. 221.

https://digitalcommons.unl.edu/physicssellmyer/221

This Article is brought to you for free and open access by the Research Papers in Physics and Astronomy at DigitalCommons@University of Nebraska - Lincoln. It has been accepted for inclusion in David Sellmyer Publications by an authorized administrator of DigitalCommons@University of Nebraska - Lincoln. 


\section{Authors}

Mircea Chipara, John R. Villarreal, Magdalena Dorina Chipara, Karen Lozano, Alin Cristian Chipara, and David J. Sellmyer 
Published in Journal of Polymer Science Part B: Polymer Physics 47:17 (2009), pp. 1644-1652; doi: 10.1002/ polb.21766

Copyright (c) 2009 Wiley Periodicals, Inc. Used by permission.

Submitted December 22, 2008; revised May 17, 2009; accepted June 5, 2009; published online July 21, 2009.

\title{
Spectroscopic investigations on Polypropylene-Carbon Nanofiber Composites: I. Raman and Electron Spin Resonance Spectroscopy
}

\author{
Mircea Chipara, ${ }_{1}^{1}$ John R. Villarreal, ${ }^{2}$ Magdalena Dorina Chipara, ${ }^{1}$ \\ Karen Lozano, ${ }^{3}$ Alin Cristian Chipara, ${ }^{2,3}$ David J. Sellmyer ${ }^{4}$ \\ ${ }^{1}$ Department of Physics and Geology, University of Texas Pan American, Edinburg, Texas 78541 \\ ${ }^{2}$ Department of Chemistry, University of Texas Pan American, Edinburg, Texas 78541 \\ ${ }^{3}$ Department of Mechanical Engineering, University of Texas Pan American, Edinburg, Texas 78541 \\ ${ }^{4}$ Department of Physics and Astronomy, University of Nebraska-Lincoln, Lincoln, Nebraska 68588 \\ Corresponding author - Mircea Chipara, Department of Physics and Geology, \\ University of Texas Pan American, Edinburg, Texas 78541; email mchipara@utpa.edu
}

\begin{abstract}
Isotactic polypropylene-vapor grown carbon nanofiber composites containing various fractions of carbon nanofibers, ranging from 0 to $20 \mathrm{wt} \%$, have been prepared. Raman spectroscopy was used to analyze the effect of the dispersion of carbon nanofibers within polypropylene and the interactions between carbon nanofibers and macromolecular chains. The as-recorded Raman spectra have been successfully fitted by a linear convolution of Lorentzian lines. Changes of the Raman lines parameters (position, intensity, width, and area) of polypropylene and carbon nanofibers were analyzed in detail. The Raman spectra of the polymeric matrix - at low concentrations of nanofibers - show important modifications that indicate strong interactions between carbon nanofibers and the polymeric matrix reflecting by vibrational dephasing of macromolecular chains. The Raman spectrum of carbon nanofibers is sensitive to the loading with carbon nanofibers, showing changes of the resonance frequencies, amplitudes, and width for both D- and G-bands. Raman data reveals the increase of the disorder, as the concentration of carbon nanofibers is increased. The presence of the typical ESR line assigned to conducting electrons delocalized over carbon nanofibers is confirmed and the presence of a spurious magnetic line due to catalyst's residues is reported.
\end{abstract}

Keywords: carbon nanotubes, ESR/EPR, nanocomposites, poly(propylene), Raman spectroscopy

\section{Introduction}

Carbon nanotubes have remarkable properties such as huge Young modulus, ${ }^{1,2}$ large strength, ${ }^{2}$ high-thermal conductivity, ${ }^{3}$ and good electrical conductivity. ${ }^{4}$ Characterized by a very high aspect ratio, ${ }^{5}$ carbon nanotubes have been perceived as excellent fillers for improved lightweight materials with enhanced structural capabilities and/or multifunctional materials. ${ }^{6,7}$ Although carbon nanofibers have remarkable physical properties (not as impressing as single and multiwalled carbon nanotubes) they are significantly cheaper providing a cost ef- 
ficient solution to polymer-based nanocomposites. ${ }^{8}$ The dispersion of carbon nanotubes and nanofibers within polymeric matrices improves the mechanical, thermal, and electrical capabilities of the pristine polymeric matrices adding eventually new properties such as electrical conductivity. ${ }^{4-8}$

This research is divided into two parts: the first focused on Raman and electron spin resonance (ESR) spectroscopy of vapor grown carbon nanofibers (VGCNF) dispersed within isotactic polypropylene (iPP), and the second concentrated on wide angle X-ray scattering (WAXS), and differential scanning calorimetry (DSC). The study aims to a better understanding of the interactions between VGCNFs and iPP adding precision and rigor to the existing literature. ${ }^{9-16}$ The second part of the study will inspect the effect of VGCNF on the crystallinity of the polymeric matrix. Preliminary investigations on the morphology and thermal stability of iPP-VGCNF, performed on the same samples, have been reported elsewhere. ${ }^{15}$ Rich and unexpected information on the interaction between VGCNF and iPP was extracted from thermogravimetric analysis (TGA) measurements, confirming the formation of an interface between iPP and VGCNF and revealing the complex structure of this interface. A thin hard layer has been tentatively identified and assigned to polymer segments captured by the nanofibers at such small distances that the van der Waals interaction between these molecules and the nanofiber is comparable with the bond energy that links covalently the molecules in the polymer. ${ }^{15}$ The presence of a soft layer that extends from the interface nanofiber - polymer at distances comparable with the radius of gyration of polypropylene was also reported. ${ }^{16}$ As TGA data suggested a more complicated structure of the interface between VGCNFs and macromolecular chains (iPP), additional investigations were initiated to obtain further experimental evidence for these findings.

The first part of this article concentrates of Raman spectroscopy investigations of iPP-VGCNF composites. This analysis is traditionally focused on the Raman lines due to the filler (VGCNFs). ${ }^{9-16}$ The original aspect of this study derives from the fact that the analysis of Raman spectra includes both the polymeric matrix and the filler. This required the computer deconvolution of the full Raman spectrum and resulted in a more accurate estimation of Raman lines positions, amplitudes, widths, and areas. The second part of this article analyzes ESR spectroscopy results. This experimental technique is not yet frequently used to investigate carbon nanotubes and carbon nanofiber composites.

Polypropylene is a macromolecule with a simple repeating unit and various tacticities defined by the position of the methyl group relative to the polymer backbone. ${ }^{17} \mathrm{iPP}$ is obtained by the addition of monomers with all substituents placed on the same side of the backbone chain. iPP is a semicrystalline polymer with a tendency to take helix like configuration (repeating unit built of three monomers related each to other by a three-fold helix axis). ${ }^{17}$ The Raman spectrum of iPP is complex, consisting of 25 parallel and polarized modes (A modes) and 52 perpendicular and depolarized (E) modes. ${ }^{18,} 19$ The analysis of Raman spectra of polypropylene is complicated by the fact that in most cases $\mathrm{A}$ and $\mathrm{E}$ modes are mixed. ${ }^{18,19}$ Certain Raman modes are not significantly affected by the configuration and conformation of macromolecular chains. For example, the Raman lines located at 1152, 1034, 900, and $841 \mathrm{~cm}^{-1}$ in molten isotactic and sindiotactic polypropylene are coincident or almost coincident. ${ }^{17-19}$ As expected, some other Raman lines are characteristic either to the sindiotactic or isotactic addition of monomers. ${ }^{17}$

Pristine polymers such as iPP show no ESR spectra. ${ }^{20}$ Under certain circumstances, residual ESR spectra may point either to catalyst residues or to trapped free radicals generated by mechanical, thermal, or oxidative processes. ${ }^{20}$ VGCNFs show ESR spectra almost identical to the ESR spectra of carbon nanotubes. ${ }^{21}$ Typically, the ESR spectrum of carbon nanotubes contains several contributions ${ }^{22-24}$; a very broad line characterized by $g>2.00$ assigned to catalyst's impurities (magnetic nanoparticles), a narrow line (sometimes asymmetric) located near $g=2.0023$ and assigned to conducting electrons delocalized over carbon nanotubes or carbon nanofibers. The ESR line originating from conducting electrons delocalized over carbon nanotubes or carbon nanofibers is temperature independent. ESR spectra may be used to assess the average orientation or carbon nanotubes/nanofibers within polymeric matrices. ${ }^{21}$ Eventually, another narrow line assigned to graphitic carbon has been reported ${ }^{22-24}$ around $g=2.0023$.

\section{Experimental Methods}

iPP-VGCNF nanocomposites were obtained by highshear mixing of iPP (Marlex HLN-120-01; Philips Sumika Polypropylene Company, density $906 \mathrm{~kg} / \mathrm{m}^{3}$; more de- 

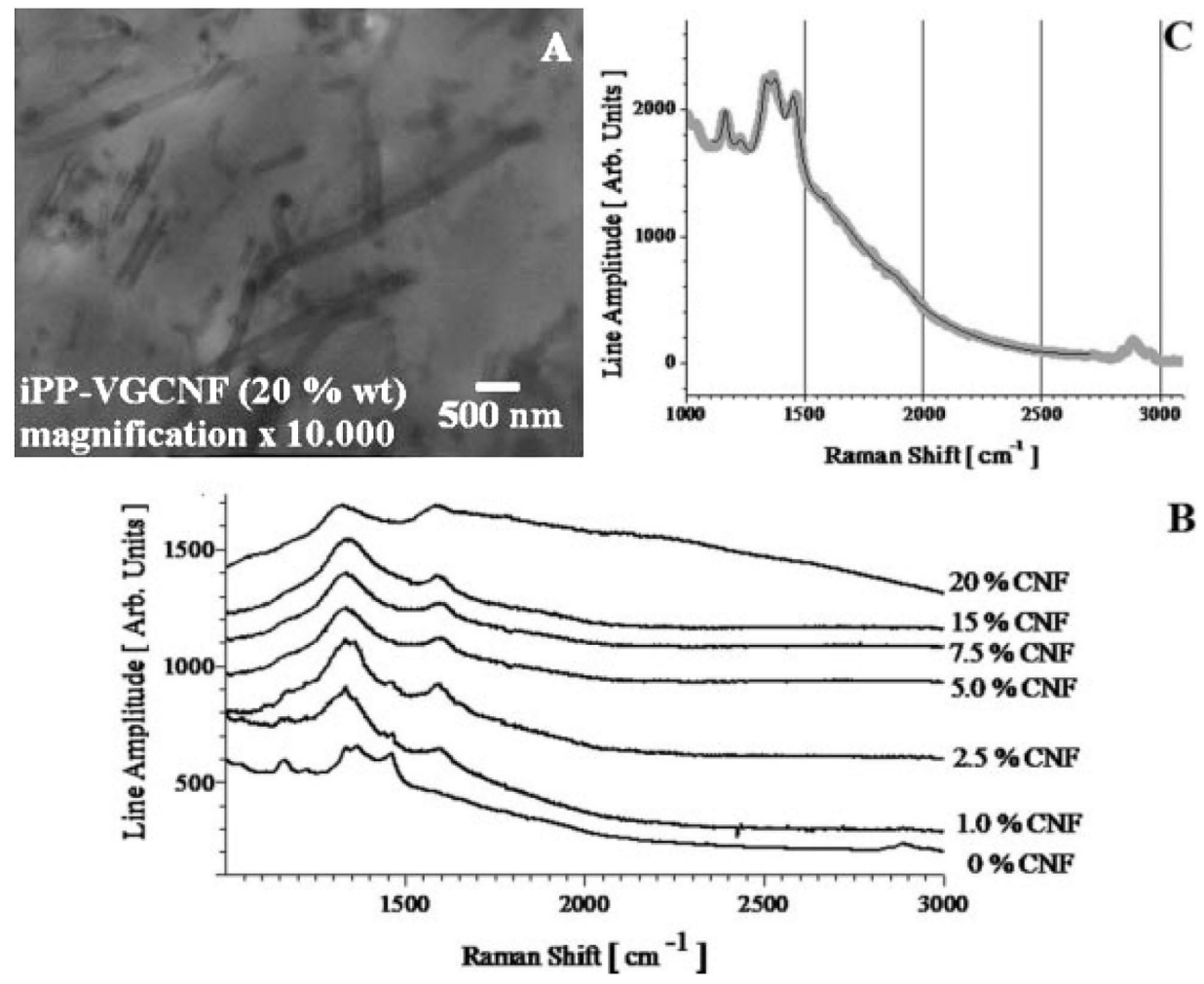

\section{B}

Figure 1. A: TEM photo of iPP-VGCNF composite; B: Raman spectra of iPP filled with various amounts of VGCNFs (expressed in wt \%); and C: The best fit of Raman spectrum in the range $1000-2800 \mathrm{~cm}^{-1}$ for the pristine polymer.

tails about the polymeric matrix are available at http:// www.cpchem.com/enu/polypropylene_tl_technical_library.asp ) with various amounts of VGCNFs (PR-24AG; Pyrograf Products, Inc.) by utilizing a HAAKE Rheomix at $65 \mathrm{rpm}$ and $180^{\circ} \mathrm{C}$ for $9 \mathrm{~min}$ followed by an additional mixing of $5 \mathrm{~min}$ at the same temperature and at $90 \mathrm{rpm}$. The as-received VGCNFs have been chemically treated as reported elsewhere ${ }^{15}$ to debundle and purify the nanofibers. Composites loaded with various amounts of VGCNFs ranging between 0 and $20 \mathrm{wt} \%$ have been prepared. Scanning electron microscopy confirmed the homogeneous distribution of CNFs within the polymeric matrix see Figure 1(A). ${ }^{15}$ Raman spectroscopy analysis has been performed by using a Bruker Sentera dispersive Raman microscope spectrometer equipped with a $785 \mathrm{~nm}$ laser diode. ESR measurements have been carried out at room temperature with a Bruker Elexsys spectrometer, operating in the X-band ( 9 GHz).

\section{Results And Discussions}

The weak intensity of the Raman lines due to polypropylene in polymer-nanotube composites has been recog- nized by several authors. ${ }^{10-13}$ This section focuses on the analysis of the Raman spectra of both VGCNF and iPP in composites containing various amounts of VGCNFs, aiming to extract new information regarding the interaction of macromolecular chains with the nanofiller from an exhaustive analysis of Raman spectra.

The Raman spectra of iPP-VGCNF, for different loadings with VGCNFs (ranging from 5 to $20 \mathrm{wt} \%$, VGCNF), are shown in Figure 1(B). The as-recorded spectra are rather complex; the contribution of iPP dominates Raman spectra of iPP-VGCNFs that contains a low concentration of VGCNFs. It is important to notice that the contributions of iPP are expected to dominate the Raman spectra characterized by shifts smaller than 1200 $\mathrm{cm}^{-1} .{ }^{19,} 25$ The Raman spectra in the range $1100-2800 \mathrm{~cm}^{-}$ ${ }^{1}$ have been simulated by a convolution of up to 11 symmetric Lorentzian lines. Figure $1(\mathrm{C})$ demonstrates the good agreement between the fitted spectrum (black and narrow line) and the as-recorded spectrum (gray and broad line).

For an exhaustive analysis of Raman lines assigned to the polymeric matrix, several domains were investigated in detail (see Figures 2 and 3). As observed from Figure 2(A), the absence of a very strong Raman line lo- 

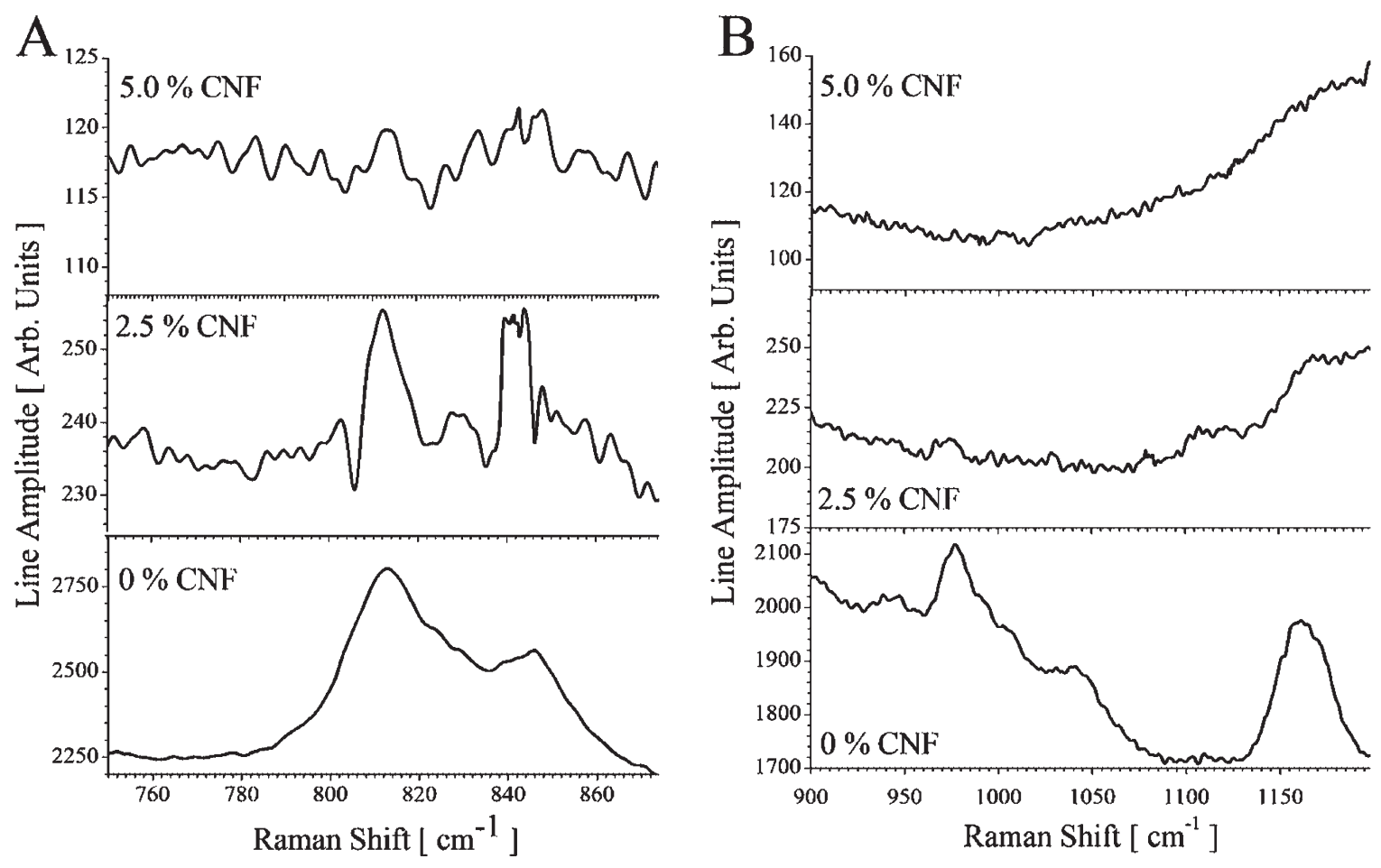

Figure 2. The Raman spectra of iPP-VGCNF composites for Raman shifts ranging between 750 and $900 \mathrm{~cm}^{-1}$ (left panel) and 900 and $1200 \mathrm{~cm}^{-1}$ (right panel), respectively.

cated at $850 \mathrm{~cm}^{-1}$ rules-out the possibility of sindiotactic-like structures. ${ }^{18,19}$ The degree of crystallinity of iPP may be inferred from Raman spectra within a threephase mode ${ }^{19}$ that implies a crystalline phase responsible for the Raman line located at $808 \mathrm{~cm}^{-1}$, a defected phase containing mostly helical chains identified by the Raman line located at $840 \mathrm{~cm}^{-1}$, and a melt-like structure assigned to amorphous polypropylene and assigned to the line noticed at $830 \mathrm{~cm}^{-1} .{ }^{19}$ As noticed from Figure 3 (bottom spectrum), the as-recorded Raman lines were observed at slightly higher Raman shifts. This may reflect the dispersive nature of these lines (the laser radiation used in this experiment had a frequency of $785 \mathrm{~nm}$, while the aforementioned line positions were reported for a laser frequency of $633 \mathrm{~nm}$ ). ${ }^{19}$ Higher concentration of VGCNFs distorts completely the polymeric matrix, up to the point at which above $5 \mathrm{wt} \%$. VGCNFs the lines of the polymeric matrix, expected to be observed in the range of 725-875 $\mathrm{cm}^{-1}$ become invisible. The Raman line located at $844 \mathrm{~cm}^{-1}$ has been assigned to short range order vibrations involving about 13 units, whereas the line located at $811 \mathrm{~cm}^{-1}$ has been assigned to short range vibrations involving 15 units. ${ }^{26}$ These lines are rapidly disappearing as the concentration of VGCNFs is increased, indicating strong distortions in the polymer structure triggered by the interaction between the polymeric matrix and VGCNFs.

As is noticed from Figure 3, the Raman line located at $2841 \mathrm{~cm}^{-1}$ assigned to $\mathrm{CH}_{2}$ symmetric stretching $(d+)$ is visible for pristine iPP but disappears completely on

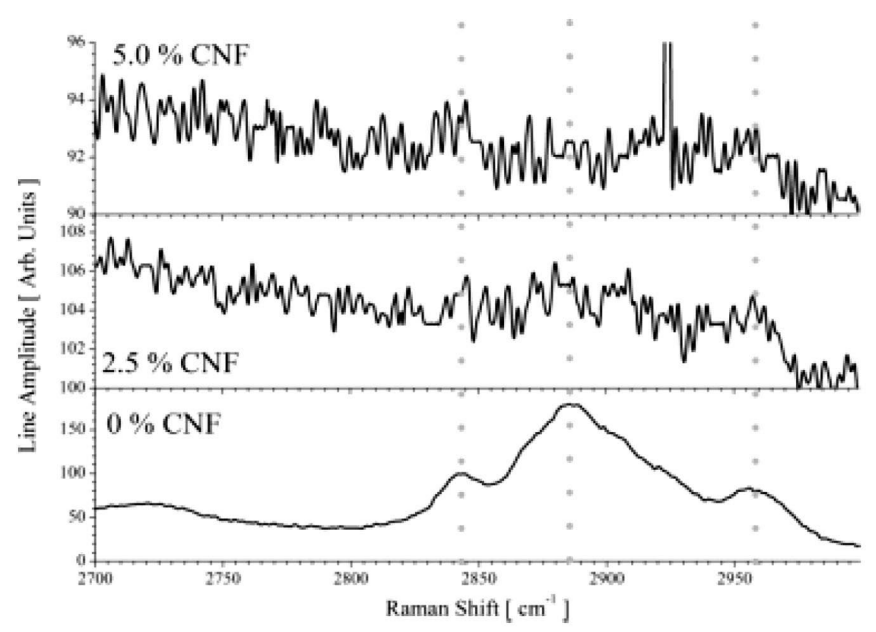

Figure 3. The Raman spectra of some iPP-VGCNF composites for Raman shifts ranging between 2700 and $3000 \mathrm{~cm}^{-1}$. 


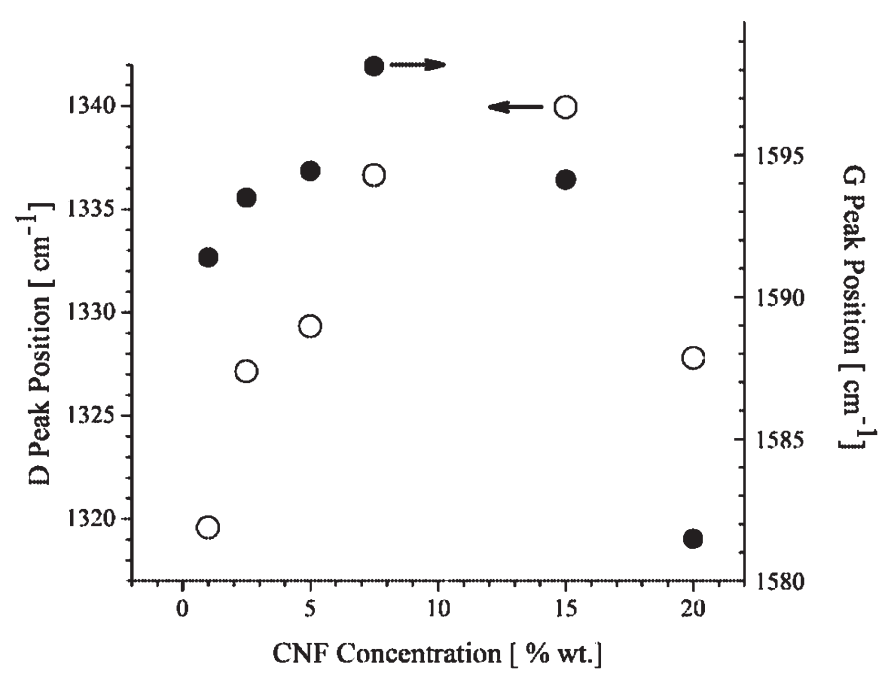

Figure 4. The dependence of the D- and G-lines position on the concentration of VGCNF.

loading with VGCNF. The Raman line assigned to $\mathrm{CH}_{2}$ asymmetric stretching $(d-)$ expected at $2920 \mathrm{~cm}^{-1}$ is significantly weaker than the symmetric one. The symmetric $\mathrm{CH}_{3}$ stretching for iPP splits into two lines, a weaker Raman line located at $2870 \mathrm{~cm}^{-1}$ (not observed in the spectrum of pristine iPP) and a stronger line located at 2883 $\mathrm{cm}^{-1}$ (see Figure 3). The asymmetric stretch $(r-)$ splits into an out of the plane stretch at $2953 \mathrm{~cm}^{-1}$ and an in-plane stretch at $2962 \mathrm{~cm}^{-1}$. In Figure 3, these line are merged into a single broad Raman line suggesting a random distribution of crystallites. From Figure 3, it is noticed that the $\mathrm{CH}_{2}$ vibrations are more affected by the loading with carbon nanofibers than the $\mathrm{CH}_{3}$ vibrations. ${ }^{19,} 26,27$ This indicates a strong interaction of VGCNFs with the polymeric backbone rather than with the $\mathrm{CH}_{3}$ side group.

The addition of VGCNF results in a sudden decrease of the intensity of all Raman lines assigned to iPP. This drop does not necessarily imply that the degree of crystallinity of the polymeric phase in iPP-VGCNF composites decreases on loading with VGCNF as the degree of crystallinity is controlled by the area of the peak rather than by its intensity. It is noticed that the width of iPP Raman lines increases very fast as VGCNFs are introduced within the polymeric matrix. Hence, even if the intensities of the Raman lines assigned to iPP are decreased below detection, the simultaneous broadening of these lines may compensate for the drop in the line intensities of Raman spectra. Under these circumstances, the load-

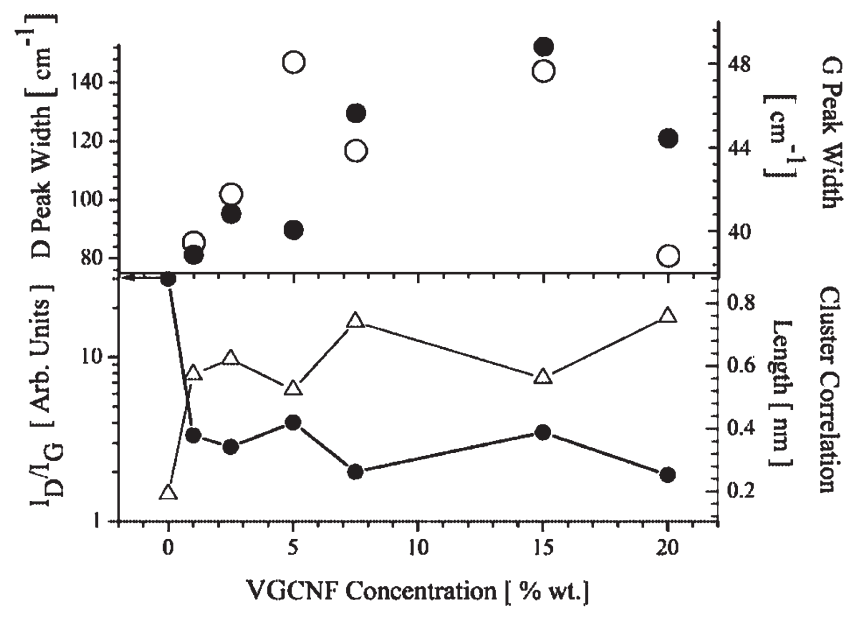

Figure 5. The effect of the concentration of VGCNF on the width of the D- and G-bands (top panel), on the ratio between the amplitude of the D- and G-bands (bottom panel, left axis) and on the estimated cluster correlation length (bottom panel, right axis).

ing of iPP with VGCNFs (up to about $2.5 \%$ VGCNFs) changes dramatically the dynamical aspects of Raman spectra confined in their line width, but do not result necessarily in a decrease of the crystallinity degree as the concentration of VGCNFs in iPP is increased. However, the observed appearance and increase of helical macromolecular chains suggests a decrease of the crystallinity degree of the iPP phase. This agrees with TGA data that demonstrated the formation of the iPP-VGCNF interface ${ }^{15}$; we are speculating that most of these chains are wrapped around VGCNFs contributing to the soft iPPVGCNF interface.

Several lines, originating from the VGCNF dispersed within the polymeric matrix were easily identified; firstorder Raman spectra include 9-16, 14, 28-32 the line located at about $1320 \mathrm{~cm}^{-1}$, identified as the D-mode and the line located at $1590 \mathrm{~cm}^{-1}$, identified as the G-mode. The D-mode is dispersive, that is, the Raman line position depends on the frequency of the laser beam. As observed from Figure 1, the D-line dominates all Raman spectra of iPPVGCNF composites suggesting that VGCNFs are disordered. As shown in Figure 4, the position of the D-line shifts toward higher wavelength as the concentration of VGCNF is increased from $0 \mathrm{wt} \%$ to about $10 \mathrm{wt} \%$. Above this concentration, the position of the D-line starts to decrease as the concentration of VGCNFs is increased. An analogous dependence has been noticed for the dependence of the width of the D-line on the loading with 
VGCNFs (see the top panel of Figure 5, left axis). The same dependence of the D-line position and amplitude has been reported in iPP loaded with single wall carbon nanotubes (SWCNTs) and assigned to the intercalation of macromolecular chains among VGCNFs. Similar data have been reported in the case of PP loaded with SWCNTs. ${ }^{10,11,30}$ The increase of the D-line position as the concentration of VGCNFs/SWCNTs is increased up to $10 \%$ was assigned to the intercalation of macromolecular chains among filler's particles, suggesting an increase of the debundling efficiency of VGCNFs during the processing step. The decrease of the D-line position as the concentration of VGCNFs/SWCNTs is further increased (above $15 \mathrm{wt} \%$ VGCNFs) was assigned to the weak aggregation of VGCNFs. ${ }^{11,12}$

The G-line is wide and intense, suggesting in-plane structural disorder. ${ }^{9-16}, 14,28-32$. Within the experimental errors neither G-band splitting nor the change in the shape of this band were noticed. ${ }^{15-17}$ This suggests that the electrical conductivity of VGCNF was not very high. The dependence of the G-line position and width is qualitatively similar to the dependence of the D-line on the Raman line position and width (see Figures 4 and 5). The ratio between the amplitude of the D- and G-lines is frequently used to characterize the degree of disorder within CNTs. As may be noticed from the right panel of Figure 5, the ratio of the amplitude of the Dand G-lines amplitude is increasing as the concentration of VGCNFs is increased, indicating a more pronounced disorder within carbon nanofibers as their concentration within the polymeric matrix is increased. The averaged in-plane crystallite size, $L$, or the so-called cluster correlation length is proportional to the ratio of the amplitudes of G- and D-lines. As observed from Figure 5, $L$ decreases as the concentration of carbon nanofibers is increased, confirming the decrease of the crystallite size (and the increase of the disorder).

Second-order Raman lines ${ }^{14,28-32}$ are also noticed (see Figure 1); the line located at about $2600 \mathrm{~cm}^{-1}$ has been assigned to the $\mathrm{D}^{*}$ mode, whereas the $2 \Gamma$ mode located about $3200 \mathrm{~cm}^{-1}$ has not been observed. The $\mathrm{D}^{\prime \prime}$ line reported in some carbon nanofibers and located at about $1500 \mathrm{~cm}^{-1}$ has not been observed. ${ }^{14,28,29,33}$ A weak line located between 1420 and $1470 \mathrm{~cm}^{-1}$, has been noticed and tentatively assigned to band modes about $\mathrm{K}$ or $\mathrm{M}$ points. ${ }^{29}$ These lines are enhanced as the loading with VGCNF is increased. However, the overlap of this line with a line due to the polymeric matrix makes difficult for a detailed analysis of these resonances.

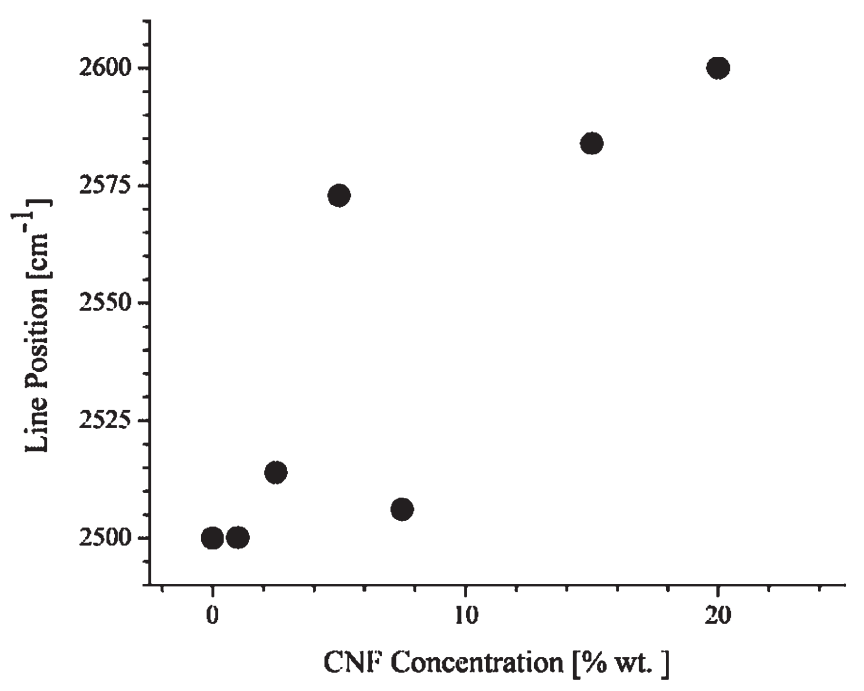

Figure 6. The effect of VGCNF concentration of the Raman line located between 2500 and $2600 \mathrm{~cm}^{-1}$.

VGCNFs exhibit broader Raman lines than SWCNTs and accordingly we were not able to deconvolute the Raman lines observed at 1549 and $1589 \mathrm{~cm}^{-1} \cdot{ }^{12}$, 13 Because of the wide distribution of VGCNFs diameters, no Raman line has been observed in the radial breathing range $\left(100-500 \mathrm{~cm}^{-1}\right)$.

Pristine polypropylene exhibits a peak located at about $2500 \mathrm{~cm}^{-1}$. By increasing the loading with carbon nanofibers this peak disappears and for carbon nanofiber concentrations in excess of $5 \% \mathrm{VGCNF}$, a peak located at about $2550 \mathrm{~cm}^{-1}$ has been noticed and assigned to the $\mathrm{D}^{*}$ mode. As may be inferred from Figure 6, the position of this peak depends on the loading with VGCNFs. Taking into account that the position of this peak may be related to the internal stresses exerted on VGCNFs, it is speculated that the VGCNFs experiences large local stresses due to the thermomechanical processing, and that these internal stresses are partially frozen within iPP-VGCNFs composites.. Accordingly, the concentration of VGCNFs is increased the local pressure increases shifting the $\mathrm{D}^{*}$ lines to higher wavenumbers (see Figure 6). ${ }^{31}$

The ESR spectra of iPP-CNF composites are shown in Figure 7. The resonance spectrum located near the $g=$ 2.00 (see the top panel of Figure 7) has been assigned to conducting electrons delocalized over VGCNFs. However, for pristine iPP, subjected to the same thermomechanical treatment a very weak ESR spectrum has been observed and assigned to free radicals generated by thermomechanical scissions of macromolecular chains during the preparation and processing of samples. ${ }^{20}$ 


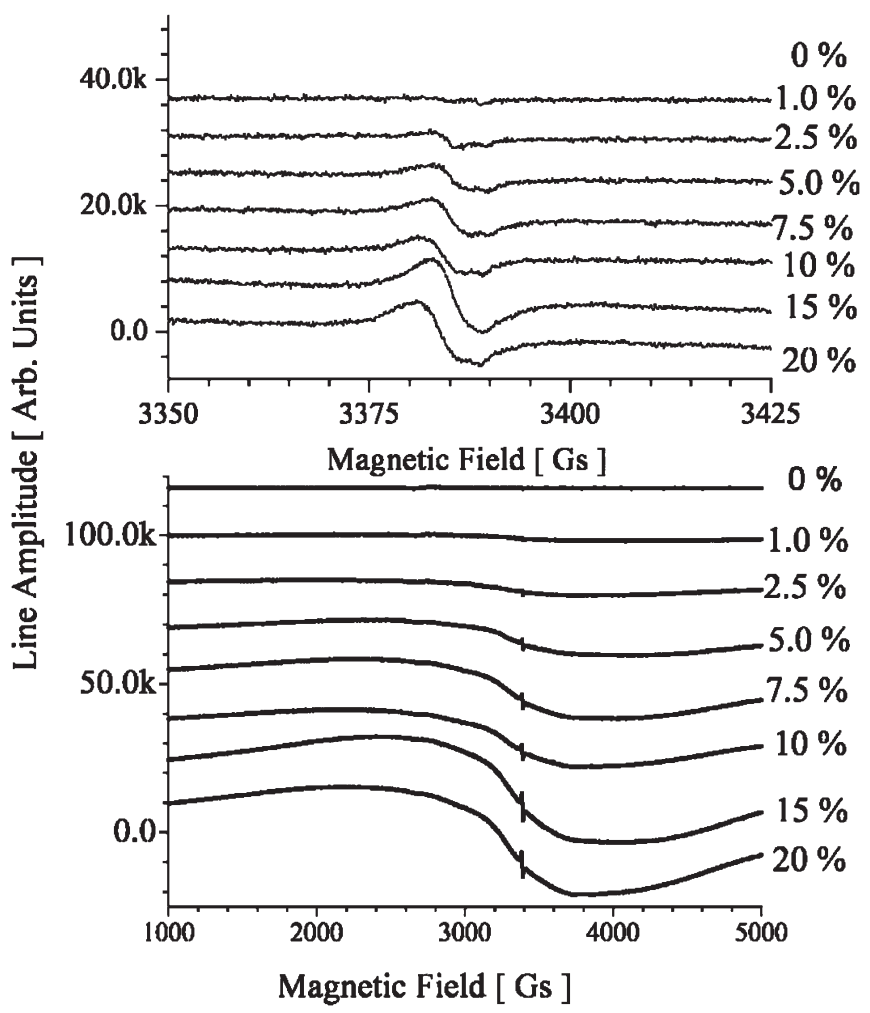

Figure 7. ESR spectra of iPP-VGCNF composites. Upper panel shows in detail the ESR spectrum of the composite around the $g=2.00$ value, whereas the bottom panel shows also the broad line due to catalyst's impurities.

The full ESR spectrum of iPP-CNF, in the X-band ( $~ 9$ $\mathrm{GHz}$ ), at room temperature is shown in the bottom panel of Figure 7. The broad and intense line originates from magnetic nanoparticles (catalyst's residues). The analysis was focused on the narrow line located close to $g=$ 2.00 and assigned to conducting electrons. ${ }^{21-24}$ This line has been successfully fitted with a single Lorentzian. For metallic nanofibers, an asymmetrical Dysonian-line resonance line shape was expected ${ }^{22}$ (due to the skin effect). The actual (symmetrical) shape of the narrow ESR line suggests that the nanofibers used to obtain these nanocomposites have a relatively low-electrical conductivity, in agreement with Raman's findings. The free-radical concentration in ESR spectroscopy is proportional to the double integral of the resonance spectrum..$^{20}$ For a simple Lorentzian line, the double integral of the resonance spectrum, is proportional to the amplitude of the ESR line and the square of the peak to peak linewidth. ${ }^{20}$, ${ }^{21}$ As it is observed from Figure 8, the intensity of the resonance line located near $g=2.00$ and the double integral of this line are increasing as the concentration of VGCNF is increased, confirming that this signal originates from VGCNF.

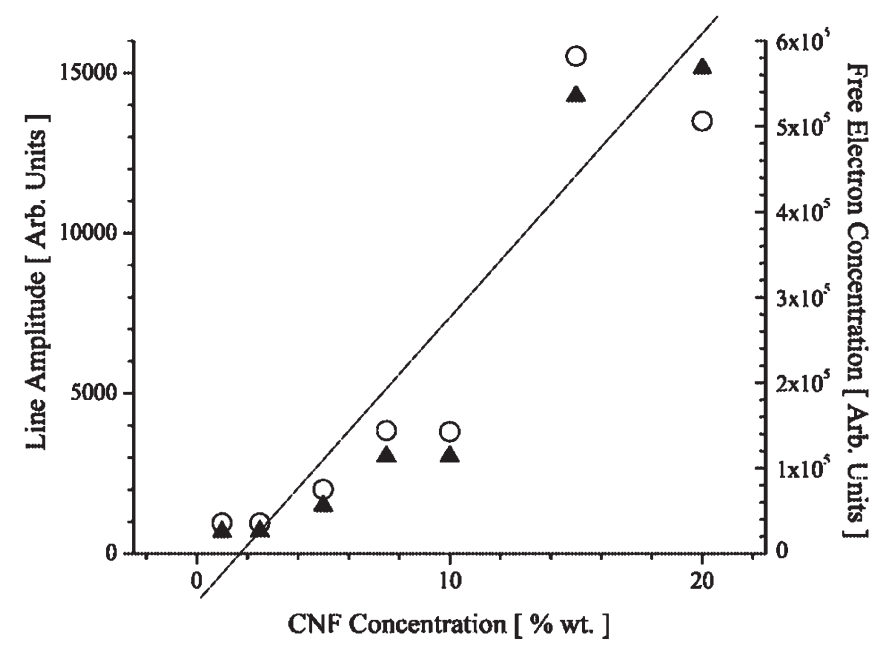

Figure 8. The dependence of the amplitude of the ESR line assigned to conducting electrons delocalized over VGCNFs on the concentration of VGCNFs (left axis) and the dependence of the concentration of free electrons on the concentration of VGCNF.

\section{Conclusions}

Both polymers and carbon nanotubes/nanofibers exhibit Raman spectra. In the case of iPP-VGCNF composites, the loading of the polymeric matrix by VGCNFs is associated with a rapid drop of the intensity of the Raman lines assigned to iPP accompanied by a fast broadening of these lines. This fast vibrational dephasing of macromolecular chain demonstrates the strong interaction between VGCNFs and iPP chains, making difficult the Raman spectroscopy study of both polymeric matrix and filler over a wide range of VGCNFs' concentrations. The loading of iPP with VGCNFs resulted in complex modifications of the Raman spectra of the polymeric matrix, which indicates the wrapping of macromolecular chains around VGCNFs and agrees with previously reported TGA data, suggesting a decrease in the overall degree of crystallinity of the polymeric matrix. ${ }^{15}$ Consequently, Raman spectroscopy was not capable to provide an accurate estimation of the degree of crystallinity of iPP-CNF composites. No evidence concerning the potential oxidation of polypropylene during the thermomechanical processing of the composites was observed from Raman studies. ${ }^{33}$ The second part of this article, will concentrate on the crystallinity of iPP-VGCNFs, as revealed by DSC and WAXS investigations.

The Raman spectra of VGCNFs show the usual dependence on the concentration of VGCNFs. An increase 
in the degree of disorder of VGCNFs as their concentration is increased was noticed from the dependence of the ratio between the amplitude of the D- and G-modes on the concentration of VGCNF. The effect of the loading with carbon nanofiber on the $\mathrm{D}^{*}$ Raman line suggests that the nanofibers are subjected to important local stresses and these stresses increase as the concentration of carbon nanofibers is increased. ${ }^{32}$ The reported changes on the D- and G-band position and width, derived from the introductions of VGCNFs are identical to the modifications reported in iPP filled with SWCNTs and multiwalled carbon nanotubes. ${ }^{9-14}$

ESR spectroscopy revealed the generation of free radicals within the pristine polymer during its processing, confirmed the presence of catalysts residues, and quantified the free electrons delocalized over the conducting domains of carbon nanofibers. Although the processing technique used to obtain iPP-VGCNFs nanocomposites resulted in free-radical generation, Raman spectroscopy ruled-out conformational and tacticity modifications of the polymeric matrix. The potential reduction of the degree of crystallinity of iPP on loading with VGCNF may express the consequence of the processing step, when the combined effect of temperature and mechanical stresses and the potential presence of oxygen traces may result in the degradation of the macromolecular chain (predominant scission reactions). The increase of the concentration of chain ends may contribute to a weak decrease of the overall crystallinity degree of iPP phase.

\section{Acknowledgments}

This work was supported by NSF-MRSEC program at the University of Nebraska, Lincoln, and by NSF grant DMR 0606224 awarded to the University of Texas Pan American. The authors thank Professor A. Rajca who allowed us to perform ESR experiments in his laboratory, within the Chemistry Department of the University of Nebraska, Lincoln.

\section{References}

1. Yu, M. F.; Lourie, O.; Dyer, M. J.; Moloni, K.; Kelly, T. F.; Ruoff, R. S. Science 2008, 287, 637-640.

2. Terrones, M.; Grobert, N.; Olivares, J.; Zhang, J. P.; Terrones, H.; Kordatos, K.; Hsu, W. K.; Hare, J. P.; Townsend, P. D.; Prassides, K.; Cheetham, A. K.; Kroto, H. W.; Walton, D. R. M. Nature 1997, 388, 52-55.
3. Che, J.; Çagin, T.; Goddard, W. A. Nanotechnology 2000, 11, 65-69.

4. Ebbesen, T. W.; Lezec, H. J.; Hiura, H.; Bennett, J. W.; Ghaemi, H. F.; Thio, T. Nature 1996, 382, 54-56.

5. Wong, S. S.; Joselevich, E.; Woolley, A. T.; Cheung, C. L.; Lieber, C. M. Nature 1998, 394, 52-55.

6. Moniruzzaman, M.; Winey, K. I. Macromolecules 2006, 39, 5194-5205.

7. Thostenson, E. K.; Ren, Z.; Chou, T. W. Compos Sci Technol 2001, 61, 1899-1912.

8. Ajayan, P. M.; Schadler, L. S.; Giannaris, C.; Rubio, A. Adv Mater 12, 750-753.

9. Bhattacharyya, A. R.; Sreekumar, T. V.; Liu, T.; Kumar, S.; Ericson, L. M.; Hauge, R. H.; Smalley, R. E. Polymer 2003, 44, 2373-2377.

10. Seo, M. K.; Lee, J. R.; Park, S. J. Mater Sci Eng A 2005, 404, 79-84.

11. Vassiliou, A.; Bikiaris, D.; Chrissafis, K.; Paraskevopoulos, K. M.; Stavrev, S. Y.; Docoslis, A. Compos Sci Technol 2008, 68, 933-943.

12. Valentini, L.; Biagiotti, J.; López-Manchado, M. A.; Santucci, S.; Kenny, J. Polym Eng Sci 2004, 44, 303-311.

13. Lee, G. W.; Jagannathan, S.; Chae, H. G.; Minus, M. L.; Kumar, S. Polymer 2008, 49, 1831-1840.

14. Valentini, L.; Biagiotti, J.; López-Manchado, M. A.; Santucci, S.; Kenny, J. Compos Sci Technol 2003, 63, 1149-1153.

15. Chipara, M. D.; Lozano, K.; Hernandez, A.; Chipara, M. T. G. A. Polym Degrad Stab 2008, 93, 871-876.

16. Zhao, Q.; Wagner, H. D. Phil Trans R Soc Lond A 2004, 362, 2407-2424.

17. Masetti, G.; Cabassi, F.; Zerbi, G. Polymer 1980, 21, 143-152.

18. Gatos, K. G.; Kandilioti, G.; Galiotis, C.; Gregoriou, V. G. Polymer 2001, 45, 4453-4464.

19. Nielsen, A. S.; Batchelder, D. N.; Pyrz, N. Polymer 2002, 43, 2671-2676.

20. Ranby, B.; Rabek, J. F. ESR Spectroscopy in Polymer Research; Springer-Verlag: Berlin, 1977.

21. Chipara, M.; Lozano, K.; Chipara, M. D. Carbon 2007, 45, 2692-2716.

22. Chipara, M.; Iacomi, F.; Zaleski, J. M.; Bai, J. B. J Optoelectron Adv Mater 2006, 8, 820-824.

23. Chipara, M.; Zaleski, J. M.; Hui, D.; Pan, N. J Polym Sci Part B: Polym Phys 2005, 43, 3406-3412.

24. Chipara, M.; Lozano, K.; Wilkins, R.; Barrera, E. V.; Pulikkathara, M. X.; Penia-Para, L.; Chipara, M. D. J Mater Sci 2008, 43, 1228-1233. 
25. Hahn, T.; Suen, W.; Kang, S.; Hsu, S. L.; Stidham, H. D.; Siedle, A. R. Polymer 2001, 42, 5813-5822.

26. Fraser, G. V.; Hendra, P. J.; Watson, D. S.; Gall, M. J.; Willis, H. A.; Cudby, M. E. A. Spectrochim Acta 2002 , 29, 1525-1533.

27. Pae, K. D.; Morrow, D. R.; Sauer, J. A. Nature 1966, 211, 514-515.

28. Bailey, R. T.; Hyde, A. J.; Kim, J. J. Spectrochim Acta 1974, 30, 91-98.

29. Masetti, G.; Cabassi, F.; Zerbi, G. Thin Solid Films 2007, 515, 2958-2964.
30. Thomsen, C. Phys Rev B 2000, 61, 4542-4544.

31. Dresselhaus, M. S. G.; Dresselhaus, G.; Jorio, A.; Souza Filho, A. G.; Pimenta, M. A.; Saito, R. Acc Chem Res 2002, 35, 1070-1078.

32. Martin Ganß, M.; Satapathy, B. K.; Thunga, M.; Weidisch, R.; Pötschke, P.; Jehnichen, D. Acta Mater 2008, 56, 2247-2261.

33. Idriss, B.; Graeme, A. G. Polym Degrad Stab 2000, 70, 269-275. 